\title{
Letter to the Editor concerning "Correlations between sedimentation sign, dural sac cross-sectional area, and clinical symptoms of degenerative lumbar spinal stenosis" by Sangbong Ko (Eur Spine J [2018] 27:1623-1628)
}

\author{
Jin Yang ${ }^{1,2} \cdot$ Qingquan Kong $^{1}$
}

Received: 10 January 2019 / Accepted: 12 May 2019 / Published online: 27 May 2019

c) Springer-Verlag GmbH Germany, part of Springer Nature 2019

\section{Dear Editor,}

With great interest, we have read the original article written by Sangbong Ko entitled "Correlations between sedimentation sign, dural sac cross-sectional area, and clinical symptoms of degenerative lumbar spinal stenosis." Overall, the authors used a very robust methodology and analyzed the correlations between the aforementioned parameters.

The author modified the classification of nerve root sedimentation sign (SedSign) [1] and subdivided positive (P) SedSign into two subtypes: $\mathrm{P}(+)$ (positive with room or empty space apparent in the dura) or $\mathrm{P}(-)$ (positive without room or no empty space in the dura). We believe that this revision has a theoretic advantage comparing to the old one to better grade SedSign.

This study found there were all statistically significant in the cross-sectional area (CSA) of all three subgroups $(p=0.01)$ and there was a correlation between SedSign and the CSA of the dural sac $(p=0.01)$. There was no correlation between pain including low back pain, referred and radiating pain and SedSign. There was no statistical significance in the correlations between the distance of the claudication and the CSA of the dura sac and the SedSign and between the functional score and the SedSign (both $p>0.05$ ).
Qingquan Kong

kqqspine@126.com

1 Department of Orthopedics Surgery, West China Hospital, Sichuan University, 37 Guoxue Lane, Chengdu 610041, Sichuan, China

2 Department of Spine Surgery, Affiliated Hospital of Southwest Medical University, 25 Taiping Road, Luzhou 646000, Sichuan, China
However, the author concluded that "Increasing severity of SedSign indicates progressively smaller dural sac CSA, but there is an inconsistent association with clinical symptoms. Therefore, it is reasonable to suggest that spinal stenosis is severe in patients with severe symptoms." In common sense, patients with radiographic severe lumbar spinal stenosis may have severe symptoms. However, multiple previous studies reported a poor correlation between stenosis on imaging and patients' symptoms or disabilities. Based on the results of this study, it is impossible to conclude that "spinal stenosis is severe in patients with severe symptoms" because no correlation between radiological parameters and clinical symptoms was found.

\section{Compliance with ethical standards}

Conflict of interest There was no conflict of interest.

Publisher's Note Springer Nature remains neutral with regard to jurisdictional claims in published maps and institutional affiliations. 